\title{
BIOMASSA E NUTRIENTES EM POVOAMENTO DE Eucalyptus dunnii Maiden NO PAMPA GAÚCHO ${ }^{1}$
}

Claudiney Couto Guimaraes ${ }^{2}$, Mauro Valdir Schumacher ${ }^{3}$, Rudi Witshoreck ${ }^{4}$, Huan Pablo Souza ${ }^{2}$ e Joel Carvalho Santo $^{5}$

\begin{abstract}
RESUMO - Este trabalho objetivou quantificar a biomassa e os nutrientes, bem como os efeitos na produtividade e no sítio, de acordo com a intensidade de colheita, em povoamento de Eucalyptus dunnii com 4 anos, em Alegrete, RS. Realizou-se um inventário da unidade amostral para a caracterização dendrométrica do povoamento. As árvores foram compartimentalizadas em raiz, madeira do tronco, casca do tronco, galhos e folhas. Amostras foram retiradas e analisadas quanto aos teores de nutrientes. A biomassa arbórea total foi de 121,9 $\mathrm{Mg} \mathrm{ha}^{-1}$, apresentando a seguinte ordem de distribuição dos componentes: madeira do tronco $>$ raiz $>$ galhos $>$ casca do tronco $>$ folhas. Em termos totais, na biomassa acima do solo, observaram-se $985 \mathrm{~kg} \mathrm{ha}^{-1}$ de macronutrientes e 32,5 $\mathrm{kg} \mathrm{ha}^{-1}$ de micronutrientes, distribuídos em: N (24\%), P (2\%), K (23\%), Ca (34\%), Mg (13\%) e S (4\%) e em B (3\%), Cu (1\%), Fe (7\%), Mn (87\%) e Zn (2\%). Entre os três cenários de colheita avaliados, Ca e $\mathrm{K}$ foram os elementos que apresentaram o maior risco para a manutenção da produtividade.
\end{abstract}

Palavras-chave: Manejo nutricional; Solos florestais; Sustentabilidade.

\section{BIOMASS AND NUTRIENTS OF Eucalyptus dunnii Maiden STAND IN PAMPA GAÚCHO}

\begin{abstract}
The study aimed to quantify biomass and nutrients as well as the effects on productivity and on site, according to the intensity of harvest in stand of Eucalyptus dunnii, with four years in Alegrete-RS. The inventory was conducted for the dendrometric characterization of the stand. The trees were compartmentalized in root, stem wood, stem bark, branches and leaves. Samples were collected and analyzed for the concentration of nutrients. The total tree biomass was $121.9 \mathrm{Mg} \mathrm{ha}^{-1}$, with the following distribution order of components: stem wood $>$ root $>$ branches $>$ stem bark $>$ leaves. In overall terms, for aboveground biomass, there was $985 \mathrm{~kg} \mathrm{ha}^{-1}$ macronutrients and 32,5 $\mathrm{kg} \mathrm{ha}^{-1}$ micronutrient, distributed: $N$ (24\%), P (2\%), K(23\%), $\mathrm{Ca}(34 \%), \mathrm{Mg}(13 \%)$ and $\mathrm{S}(4 \%)$ and $\mathrm{B}(3 \%), \mathrm{Cu}(1 \%), \mathrm{Fe}(7 \%), \mathrm{Mn}(87 \%)$ and $\mathrm{Zn}(2 \%)$. Among the three harvesting scenarios evaluated $C a$ and $K$ were the elements that showed the greatest risk to the maintenance of productivity.
\end{abstract}

Keywords: Nutritional management; Forest soils; Sustainability.

\footnotetext{
${ }^{1}$ Recebido em 11.12.2013 aceito para publicação em 18.06.2015.

${ }^{2}$ Stora Enso Florestal RS, Rosário do Sul, RS - Brasil. E-mail: <claudiney.couto@hotmail.com>e <huan.souza@hotmail.com>. ${ }^{3}$ Universidade Federal de Santa Maria, Departamento de Ciências Florestais, Santa Maria, RS - Brasil. Email<mvschumacher@gmail.com>. ${ }^{4}$ Universidade Federal de Santa Maria, Doutorado em Engenharia Florestal, Santa Maria, RS - Brasil. Email: $<$ rwitshoreck@yahoo.com.br $>$ ${ }^{5}$ Universidade Federal de Santa Maria, Graduando em Engenharia Florestal, Santa Maria, RS - Brasil. Email: <joelufsm@gmail.com>.
} 


\section{INTRODUÇÃO}

As plantações com o gênero Eucalyptus têm sido estabelecidas na região Oeste do Rio Grande do Sul, em escala apresentando índices competitivos de produtividade em relação às demais regiões do Brasil. Segundo o Anuário Estatístico 2013 da Associação Brasileira de Florestas (ABRAF), o Estado do Rio Grande do Sul contabilizou, em 2012, 284,71 mil hectares cultivados com eucalipto.

As espécies do gênero Eucalyptus apresentam tolerância a altas concentrações de alumínio na solução do solo (SILVA et al., 2004; POSCHENRIEDER et al., 2008; TAHARA et al., 2008), à baixa fertilidade natural e a níveis de cálcio e magnésio inferiores aos estabelecidos como críticos para a maioria das culturas (BARROS; NOVAIS, 1990; RIBEIRO et al., 1999). Os mecanismos de tolerância ao Al são vários, podendo ser fisiológicos (SILVA et al., 2004; POSCHENRIEDER et al., 2008) e, ou, indiretos, pela complexação do Al pela fração orgânica (VIEIRA, et al., 2008).

A produção de biomassa pode variar intensamente conforme a disponibilidade de recursos do sítio florestal, que influenciam na fotossíntese, na compartimentalização do carbono, na produção de folhas, na respiração, entre outros (RYAN et al., 2010). Além desses aspectos, através da análise da dinâmica dos processos da ciclagem, podem-se obter informações sobre a distribuição de nutrientes no ecossistema, que permitirão inferir sobre os fluxos entre os diferentes compartimentos da floresta (SCHUMACHER; POGGIANI, 2003; VITAL et al., 2004; VIEIRA; SCHUMACHER, 2010).

A manutenção da capacidade produtiva de um sítio só poderá ser mantida em longo prazo, quando a utilização da biomassa e as perdas pela erosão forem repostas de forma eficiente, sendo fundamental quantificar a biomassa produzida e a quantidade de nutrientes que é exportada pela colheita florestal (SCHUMACHER; CALDEIRA, 2001).

Eucalyptus dunnii ocorre naturalmente no Nordeste de New South Wales e Sudeste de Queensland, na Austrália. A média das temperaturas máximas do mês mais quente está entre 27 e $30^{\circ} \mathrm{C}$, e a média das mínimas do mês mais frio varia de 0 a $3{ }^{\circ} \mathrm{C}$. A precipitação pluviométrica média anual oscila de 845 a $1950 \mathrm{~mm}$. A árvore pode atingir $50 \mathrm{~m}$ de altura, com diâmetro à altura do peito (DAP) entre $1 \mathrm{e} 1,5 \mathrm{~m}$ (ocasionalmente 2,5 m) (HIGA, 1998; JOVANOVIC et al., 2000).

O objetivo deste trabalho foi quantificar a biomassa e os nutrientes, bem como os efeitos na produtividade e no sítio, de acordo com a intensidade de colheita, em um povoamento de Eucalyptus dunnii.

\section{MATERIAL E MÉTODOS}

\subsection{Caracterização do sítio}

Este estudo foi realizado em um povoamento implantado com mudas seminais de Eucalyptus dunnii, localizado na Fazenda Chica Barbosa, no Município de Alegrete, RS. Aárea pertence à StoraEnso Florestal-RS, com coordenadas geográficas centrais: $55^{\circ} 34^{\prime} 38^{\prime \prime}$ de latitude Oeste e $29^{\circ} 46^{\prime} 43^{\prime \prime}$ de latitude Sul.

O clima da região, de acordo com a classificação apresentada por Maluf (2000), é considerado subtemperado úmido, com geadas de maio a agosto, e calor intenso, principalmente nos meses de janeiro e fevereiro, sendo a temperatura média do mês mais quente $>22{ }^{\circ} \mathrm{C}$ e temperatura média anual $>18^{\circ} \mathrm{C}$. A precipitação apresenta índices pluviométricos variando de 1.250 a $1.500 \mathrm{~mm}$.

O solo da área do estudo é classificado como Argissolo Vermelho distrófico típico (EMBRAPA, 2006). De acordo com Streck et al. (2008), o solo apresenta baixa fertilidade natural. Os atributos físicos e químicos são apresentados na Tabela 1 .

\subsection{O povoamento de Eucalytpus dunnii}

O plantio foi realizado em outubro de 2007, com densidade inicial de 1.428 plantas por hectare. Para a implantação, realizou-se a subsolagem em setembro de 2007, utilizando subsolador com três hastes, incorporando $300 \mathrm{~kg} \mathrm{ha}^{-1}$ de fosfato natural reativo (GAFSA, 12\% $\mathrm{P}_{2} \mathrm{O}_{5}$ solúvel em ácido cítrico) no centro e a $40 \mathrm{~cm}$ de profundidade, seguido de gradagem leve. Foram realizadas três fertilizações pós-plantio, sendo a primeira 15 dias após o plantio, utilizando a fórmula $\mathrm{N}-\mathrm{P}_{2} \mathrm{O}_{5}-\mathrm{K}_{2} \mathrm{O}$ de 06-30-06 +0,6\% Bo, 110 g planta $^{-1}$ (126,5 $\left.\mathrm{kg} \mathrm{ha}^{-1}\right)$ dividida em duas subdoses de $55 \mathrm{~g}$ incorporadas a $15 \mathrm{~cm}$ de cada lado da muda. A segunda adubação foi realizada aos 90 dias pós-plantio, utilizando a fórmula 20-05-20 + 0,2\% Bo $+0,4 \% \mathrm{Zn}$, $122 \mathrm{~g} \mathrm{planta}^{-1}\left(140 \mathrm{~kg} \mathrm{ha}^{-1}\right)$ aplicada manualmente na projeção da copa. A terceira, aos 270 dias, utilizando 
Tabela 1 - Atributos físicos e químicos do solo na área implantada com Eucalyptus dunnii aos 4 anos de idade. Table 1 - Physical and chemical attributes of the soil in the area implanted with Eucalyptus dunnii at 4 years of age.

\begin{tabular}{|c|c|c|c|c|c|c|c|c|c|}
\hline \multirow{2}{*}{$\begin{array}{l}\text { Prof } \\
(\mathrm{cm})\end{array}$} & \multirow{2}{*}{$\begin{array}{l}\text { Densidade } \\
\mathrm{g} \mathrm{cm}^{3}\end{array}$} & \multicolumn{4}{|c|}{ Distribuição das partículas \% } & \multirow{2}{*}{$\begin{array}{c}\mathrm{MO} \\
\%\end{array}$} & \multirow{2}{*}{$\begin{array}{c}\mathrm{pH} \\
\left(\mathrm{H}_{2} \mathrm{O}\right)\end{array}$} & \multirow{2}{*}{$\begin{array}{c}\mathrm{CTC}_{\text {efet. }} \\
\mathrm{cmol}_{\mathrm{c}} \mathrm{dm}^{-3}\end{array}$} & \multirow[t]{2}{*}{$\mathrm{Ca}$} \\
\hline & & Areia Grossa & Areia Fina & Silte & Argila & & & & \\
\hline $0-20$ & 1,5 & 30 & 25 & 26 & 19 & 1,4 & 4,4 & 3,4 & 0,9 \\
\hline $20-40$ & 1,5 & 37 & 16 & 28 & 19 & 1,3 & 4,4 & 4,8 & 1,6 \\
\hline $40-100$ & 1,5 & 24 & 15 & 27 & 33 & 1,1 & 4,6 & 5,5 & 2,8 \\
\hline \multirow{2}{*}{$\begin{array}{l}\text { Prof } \\
(\mathrm{cm})\end{array}$} & $\mathrm{Mg}$ & $\mathrm{P}$ & $\mathrm{K}$ & $\mathrm{S}$ & B & $\mathrm{Cu}$ & $\mathrm{Zn}$ & $\mathrm{V}$ & $\mathrm{m}$ \\
\hline & $\mathrm{cmol}_{\mathrm{c}} \mathrm{dm}^{-3}$ & \multicolumn{4}{|c|}{$\mathrm{mg} \mathrm{dm}^{-3}$} & & & \multicolumn{2}{|c|}{$\%$} \\
\hline $0-20$ & 1,0 & 2,3 & 38,7 & 5,3 & 0,3 & 5 & 3,2 & 15 & 41 \\
\hline $20-40$ & 0,7 & 0,8 & 20,7 & 6,3 & 0,2 & 7 & 1,5 & 11 & 52 \\
\hline $40-100$ & 0,9 & 0,6 & 12,7 & 6 & 0,3 & 7 & 1 & 20 & 33 \\
\hline
\end{tabular}

a fórmula $22-00-18+1 \% \mathrm{~S}+0,3 \% \mathrm{~B}, 122 \mathrm{~g} \mathrm{planta}^{-1}$ $\left(140 \mathrm{~kg} \mathrm{ha}^{-1}\right)$ aplicada mecanicamente na entrelinha. Em nenhum momento foi realizada a calagem.

Os dados foram coletados em outubro de 2011, quando o povoamento se encontrava com 4 anos de idade. Inicialmente, realizou-se um inventário em uma área de 10 hectares, com o objetivo de caracterizar o povoamento quanto às variáveis dendrométricas. Verificou-se, em termos médios, um diâmetro à altura do peito (DAP) de 13,5 cm e altura total de 15,3 m. O incremento médio anual com casca (IMA cc) observado foi de $27 \mathrm{~m}^{3} \mathrm{ha}^{-1}$.

\subsection{Metodologia usada na avaliação da biomassa e estoque de nutrientes}

Para determinação da biomassa acima do solo e raízes, utilizou-se a metodologia proposta por Neves (2000), em que foram selecionadas três árvores por parcela do inventário, totalizando nove árvores, sendo escolhidas pelo DAP médio - desvio-padrão, DAP médio e DAP médio + desvio-padrão. As árvores selecionadas foram seccionadas ao nível do solo e fracionadas nos seguintes componentes: folha, galho, casca do tronco, madeira do tronco e raiz. A biomassa de cada componente foi determinada através da pesagem com balança de gancho. Coletaram-se amostras (150 g cada) dos diferentes componentes, sendo uma amostra por árvore (de forma aleatória) dos componentes folha e galho (galhos finos e grossos, vivos e mortos localizados em toda a copa). Para madeira e casca foram coletadas três amostras por árvore distribuídas ao longo do fuste comercial, nas posições medianas das secções resultantes da divisão em três partes iguais.
Para obtenção da biomassa das raízes foram selecionadas três árvores entre as nove utilizadas para a biomassa acima do solo em cada povoamento, sendo elas escolhidas pelo DAP médio - desvio-padrão, DAP médio e DAP médio + desvio-padrão. O sistema radicular (toco e raízes grossas) das árvores foi extraído por retroescavadeira e escavação manual (pás e enxadas), na área útil da árvore média de cada parcela até a profundidade de $1 \mathrm{~m}$. O solo foi espalhado sobre lona plástica, da qual foram retiradas manualmente as raízes, pesadas na sua totalidade e extraída uma amostra. Todas as amostras foram pesadas no campo com balança de precisão, colocadas em embalagens de papel e devidamente identificadas.

Para calcular a biomassa por hectare, a massa de cada nutriente nos componentes das árvores e o estoque de nutrientes na biomassa por hectare, utilizou-se a metodologia descrita por Santana et al. (2008a), em que a estimativa da biomassa por hectare foi determinada através da extrapolação com base na biomassa seca de cada componente por unidade amostral. A massa de cada nutriente nos componentes das árvores (casca do tronco, madeira do tronco, folha, galho e raiz) foi obtida através do produto entre a biomassa seca e as concentrações de nutrientes em cada um dos referidos componentes, enquanto a estimativa do estoque de nutrientes na biomassa por hectare pela extrapolação do estoque médio de nutrientes por compartimento e com base em cada unidade amostral.

A coleta do solo para as análises química e física foi realizada nas trincheiras, de onde foram retiradas as raízes, nas camadas de 0-20, 20-40 e 40-100 cm, de acordo com a metodologia proposta pela Embrapa (1997). 


\subsection{Análises químicas das amostras}

As análises dos tecidos e do solo foram realizadas no Laboratório de Ecologia Florestal do Departamento de Engenharia Florestal da Universidade Federal de Santa Maria (UFSM), seguindo a metodologia de Tedesco et al. (1995), preconizada pela Comissão de Química e Fertilidade do Solo - RS/SC (2004).

\subsection{Estimativa da remoção de nutrientes através da colheita da biomassa}

A remoção de nutrientes através da colheita da biomassa foi estimada considerando três cenários: $1^{\circ}$ ) Colheita de toda a árvore, $2^{\circ}$ ) Colheita da madeira com casca e $3^{\circ}$ ) Colheita da madeira.

\subsection{Procedimentos estatísticos}

As médias dos teores de nutrientes foram comparadas pelo teste de Tukey a $5 \%$ de probabilidade de erro, com o uso do aplicativo SAS (2003). Considerou-se na análise estatística um delineamento inteiramente casualizado, tendo como tratamentos os teores de nutrientes (N, P, K, Ca, Mg, S, B, Cu, Fe, Mn e Zn) em cada componente da biomassa (folha, galho, casca do tronco, madeira do tronco e raiz) e respectivos números de repetição (árvores amostradas). No caso da casca e madeira do tronco, foi utilizada a média das três subamostras coletadas ao longo do tronco, totalizando nove amostras em cada componente.

\section{RESULTADOS}

\subsection{Biomassa acumulada e estoque de nutrientes}

A produção de biomassa foi de $121,9 \mathrm{Mg} \mathrm{ha}^{-1} \mathrm{e}$ apresentou a seguinte ordem de distribuição e partição relativa: madeira do tronco $(63 \%)>\operatorname{raiz}(14 \%)>$ galho $(11 \%)>$ casca do tronco $(8 \%)>$ folha $(4 \%)$. Considerando apenas a biomassa acima do solo, verificou-se uma produção de $104,5 \mathrm{Mg} \mathrm{ha}^{-1}$.

A distribuição dos estoques de nutrientes na biomassa arbórea é apresentada na Figura 1 .

Quanto aos teores dos nutrientes nos componentes da biomassa (Tabela 2), houve diferenciação estatística $(\mathrm{P}<0.05)$.

A magnitude de concentração dos macronutrientes apresentou, em ordem decrescente, a seguinte tendência de distribuição: folhas $>$ casca do tronco $>$ galhos $>$ raiz $>$ madeira do tronco e para os micronutrientes: raiz $>$ folhas $>$ casca do tronco $>$ galhos $>$ madeira do tronco. Destaca-se que, considerando apenas a biomassa acima do solo, a componente folha apresentou a maior concentração para a maioria dos elementos.

Com relação às quantidades totais de nutrientes na biomassa (Tabela 3), observou-se acúmulo de 1.213 $\mathrm{kg} \mathrm{ha}^{-1}$ e $61 \mathrm{~kg} \mathrm{ha}^{-1}$ de macro e micronutrientes, respectivamente.

Para a biomassa acima do solo, obtiveram-se 985 $\mathrm{kg} \mathrm{ha}^{-1}$ de macronutrientes, distribuídos em: N (24\%), $\mathrm{P}(2 \%), \mathrm{K}(23 \%), \mathrm{Ca}(34 \%), \mathrm{Mg}(13 \%)$ e S (4\%) e 32,5 $\mathrm{kg} \mathrm{ha}^{-1}$ de micronutrientes, distribuídos em: B (3\%), $\mathrm{Cu}(1 \%), \mathrm{Fe}(7 \%), \mathrm{Mn}(87 \%)$ e $\mathrm{Zn}(2 \%)$, com a seguinte ordem de distribuição dos elementos $\mathrm{Ca}>\mathrm{N}>\mathrm{K}>$ $\mathrm{Mg}>\mathrm{S}>\mathrm{P}$ e $\mathrm{Mn}>\mathrm{Fe}>\mathrm{B}>\mathrm{Zn}>\mathrm{Cu}$.

Entre os micronutrientes, o Fe apresentou o maior teor na raiz. Na madeira do tronco, concentraram-se os maiores teores de $\mathrm{Cu}, \mathrm{B}$ e $\mathrm{Zn}$, enquanto na casca do tronco o maior teor de $\mathrm{Mn}$.

\subsection{Remoção de nutrientes pela colheita da biomassa}

Na Tabela 4 são apresentadas as estimativas de remoção dos nutrientes de acordo com três cenários de colheita da biomassa propostos.

A retirada apenas da madeira proporcionou a menor remoção de nutrientes do sistema, com manutenção dos nutrientes acumulados nos demais componentes da biomassa em relação à colheita da madeira com casca de: $69 \%$ N, 70\% P, 56\% K, 89\% Ca, Mg 61\%, S 39\% eB $50 \%, \mathrm{Cu} 55 \%, \mathrm{Fe} 43 \%$, Mn 88\% e Zn 55\%. Considerando a colheita de toda a árvore, essa permanência decresce para: $31 \% \mathrm{~N}, 46 \% \mathrm{P}, 27 \% \mathrm{~K}, 83 \% \mathrm{Ca}, \mathrm{Mg} 46 \%$, S $16 \%$ e B $23 \%$, Cu $24 \%$, Fe $13 \%$, Mn $80 \%$ e Zn $24 \%$.

Portanto, de acordo com a intensidade da colheita da biomassa, pode-se definir o seguinte gradiente de remoção dos nutrientes: colheita de toda a árvore $\mathrm{Ca}$ $>\mathrm{N}>\mathrm{K}>\mathrm{Mg}>\mathrm{S}>\mathrm{P}$; madeira com casca $\mathrm{Ca}>\mathrm{K}>$ $\mathrm{N}>\mathrm{Mg}>\mathrm{S}>\mathrm{P}$; e apenas a madeira do tronco: $\mathrm{K}>$ $\mathrm{N}>\mathrm{Mg}>\mathrm{Ca}>\mathrm{S}>\mathrm{P}$ e $\mathrm{Mn}>\mathrm{Fe}>\mathrm{B}>\mathrm{Zn}>\mathrm{Cu}$, nos três cenários avaliados.

\section{DISCUSSÃO}

\subsection{Biomassa acumulada e estoque de nutrientes}

A produção de biomassa acima do solo, verificada neste estudo, foi similar à encontrada por Santana et 


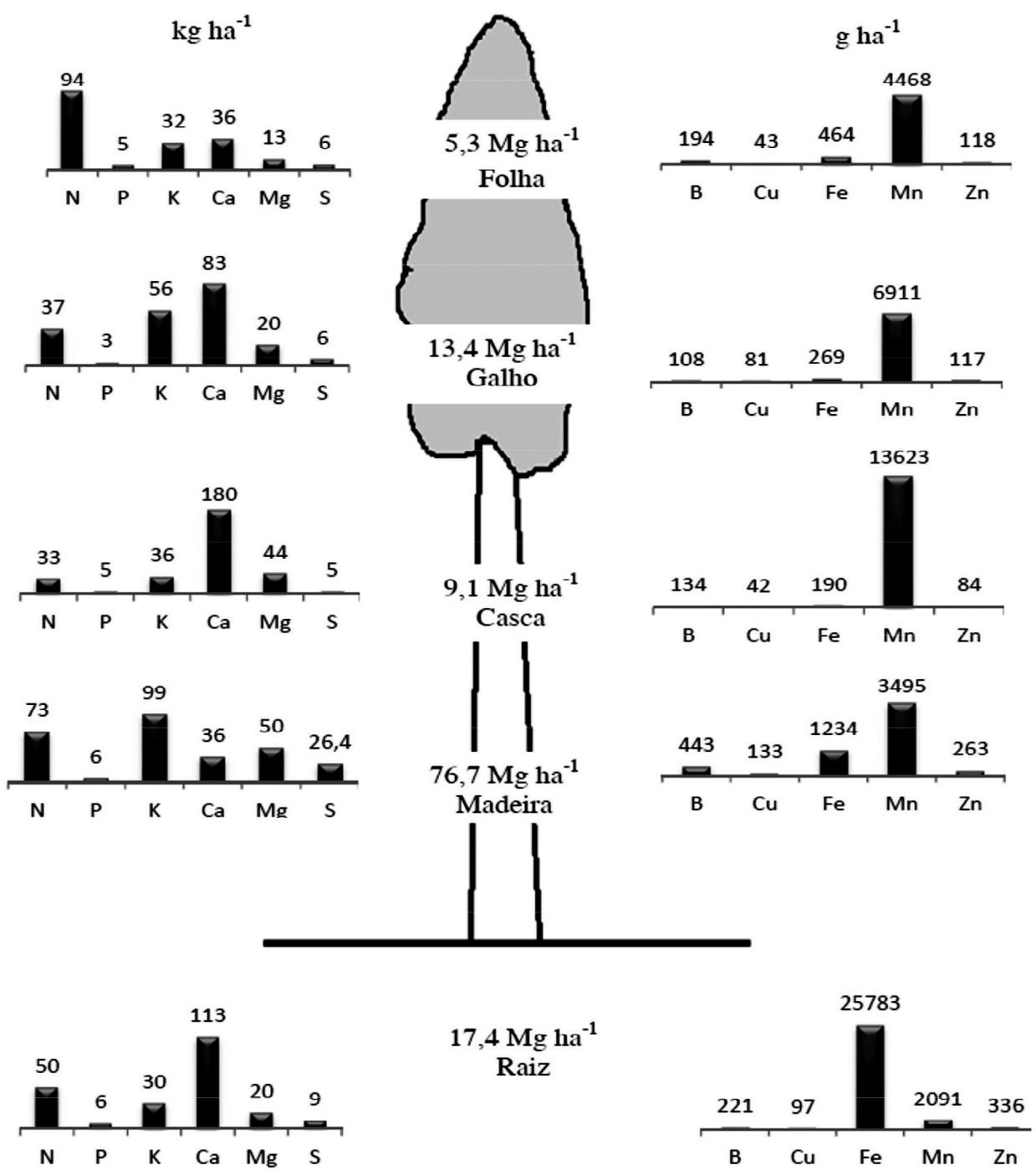

Figura 1 - Estoque de nutrientes na biomassa arbórea do Eucalyptus dunnii aos 4 anos de idade.

Figure 1 - Nutrients stock in tree biomass for Eucalyptus dunnii at 4 years of age.

Tabela 2 - Teores médios dos nutrientes na biomassa por componente do Eucalyptus dunnii aos 4 anos de idade.

Table 2 -Mean content of the nutrients in biomass by component of Eucalyptus dunnii at 4 years of age.

\begin{tabular}{|c|c|c|c|c|c|c|c|c|c|c|c|}
\hline \multirow{2}{*}{ Componente } & \multicolumn{6}{|c|}{ Macronutrientes $\left(\mathrm{g} \mathrm{kg}^{-1}\right)$} & \multicolumn{5}{|c|}{ Micronutrientes $\left(\mathrm{mg} \mathrm{kg}^{-1}\right)$} \\
\hline & $\mathrm{N}$ & $\mathrm{P}$ & $\mathrm{K}$ & $\mathrm{Ca}$ & $\mathrm{Mg}$ & $\mathrm{S}$ & $\mathrm{B}$ & $\mathrm{Cu}$ & $\mathrm{Fe}$ & $\mathrm{Mn}$ & $\mathrm{Zn}$ \\
\hline $\mathrm{Mt}^{11}$ & $1,0 \mathrm{c}^{12}$ & $0,1 \mathrm{~d}$ & $1,3 \mathrm{c}$ & $0,5 \mathrm{c}$ & $0,7 \mathrm{~d}$ & $0,3 \mathrm{c}$ & $5,7 \mathrm{~d}$ & $1,7 \mathrm{c}$ & $16,2 b$ & $46,3 \mathrm{~d}$ & $3,4 \mathrm{~b}$ \\
\hline $\mathrm{Ct}$ & $3,6 b$ & $0,6 \mathrm{~b}$ & $4,1 \mathrm{~b}$ & $19,2 \mathrm{a}$ & $4,8 \mathrm{a}$ & $0,5 b$ & $14,5 b$ & $4,6 b$ & $19,5 b$ & $1403,1 \mathrm{a}$ & $9,2 \mathrm{~b}$ \\
\hline $\mathrm{F}$ & $17,9 \mathrm{a}$ & $1,0 \mathrm{a}$ & $5,9 \mathrm{a}$ & $6,7 b$ & $2,5 b$ & $1,2 \mathrm{a}$ & $36,1 \mathrm{a}$ & $8,4 \mathrm{a}$ & $86,2 b$ & $809,9 b$ & $23,0 \mathrm{a}$ \\
\hline G & $2,8 \mathrm{~b}$ & $0,2 \mathrm{~cd}$ & $4,4 b$ & $6,2 b$ & $1,5 \mathrm{c}$ & $0,5 b$ & $8,2 \mathrm{~cd}$ & $6,3 b$ & $20,9 b$ & $521,3 \mathrm{c}$ & $8,6 b$ \\
\hline $\mathrm{R}$ & $2,8 b$ & $0,3 \mathrm{c}$ & $1,7 \mathrm{c}$ & $6,4 \mathrm{~b}$ & $1,2 \mathrm{~cd}$ & $0,5 b$ & $12,0 \mathrm{bc}$ & $5,4 \mathrm{~b}$ & $1351,4 \mathrm{a}$ & $122,3 \mathrm{~d}$ & $19,1 \mathrm{a}$ \\
\hline
\end{tabular}

${ }^{1} \mathrm{Mt}=$ madeira do tronco; $\mathrm{Ct}=$ casca do tronco; $\mathrm{F}=$ folhas; $\mathrm{G}=$ galhos; $\mathrm{R}=$ raiz; ${ }^{12}$ Letras iguais na vertical, não diferem estatisticamente entre os nutrientes e seus respectivos componentes da biomassa, ao nível de 0,05 de significância, pelo teste de Tukey. 
Tabela 3 - Quantidade de nutrientes da biomassa por componente de Eucalyptus dunnii aos 4 anos de idade. Table 3 - Nutrient amounts of biomass by component of Eucalyptus dunnii at 4 years of age.

\begin{tabular}{|c|c|c|c|c|c|c|c|c|c|c|c|}
\hline \multirow{2}{*}{ Comp } & \multicolumn{6}{|c|}{ Macronutrientes $\left(\mathrm{kg} \mathrm{ha}^{-1}\right)$} & \multicolumn{5}{|c|}{ Micronutrientes $\left(\mathrm{g} \mathrm{ha}^{-1}\right)$} \\
\hline & $\mathrm{N}$ & $\mathrm{P}$ & $\mathrm{K}$ & $\mathrm{Ca}$ & $\mathrm{Mg}$ & $\mathrm{S}$ & $\mathrm{B}$ & $\mathrm{Cu}$ & $\mathrm{Fe}$ & $\mathrm{Mn}$ & $\mathrm{Zn}$ \\
\hline $\mathrm{Mt}^{\mathrm{1}}$ & $\begin{array}{c}73,07 \\
(25,50)^{12}\end{array}$ & $\begin{array}{c}6,10 \\
(24,12)\end{array}$ & $\begin{array}{c}98,77 \\
(39,03)\end{array}$ & $\begin{array}{l}36,36 \\
(8,10)\end{array}$ & $\begin{array}{c}49,64 \\
(33,88)\end{array}$ & $\begin{array}{c}26,39 \\
(49,97)\end{array}$ & $\begin{array}{l}442,82 \\
(40,27)\end{array}$ & $\begin{array}{l}132,57 \\
(33,45)\end{array}$ & $\begin{array}{c}1234,18 \\
(4,42)\end{array}$ & $\begin{array}{c}3494,59 \\
(11,42)\end{array}$ & $\begin{array}{l}262,98 \\
(28,65)\end{array}$ \\
\hline$\dot{o}^{13}$ & 21,74 & 3,45 & 28,45 & 18,97 & 13,40 & 5,08 & 101,06 & 30,36 & 349,06 & 1076,57 & 80,97 \\
\hline $\mathrm{CV}^{4}$ & 29,75 & 56,48 & 28,80 & 52,17 & 27,00 & 19,26 & 170,01 & 22,90 & 28,28 & 30,81 & 30,79 \\
\hline $\mathrm{Ct}$ & $\begin{array}{c}32,73 \\
(11,43)\end{array}$ & $\begin{array}{c}5,24 \\
(20,72)\end{array}$ & $\begin{array}{c}35,81 \\
(14,15)\end{array}$ & $\begin{array}{l}179,85 \\
(40,05)\end{array}$ & $\begin{array}{c}43,78 \\
(29,88)\end{array}$ & $\begin{array}{c}4,67 \\
(8,84)\end{array}$ & $\begin{array}{l}134,01 \\
(12,19)\end{array}$ & $\begin{array}{c}42,10 \\
(10,62)\end{array}$ & $\begin{array}{c}189,77 \\
(0,68)\end{array}$ & $\begin{array}{c}13623,17 \\
(44,54)\end{array}$ & $\begin{array}{l}84,41 \\
(9,20)\end{array}$ \\
\hline ó & 11,01 & 1,28 & 11,76 & 97,72 & 14,25 & 1,57 & 53,07 & 15,37 & 125,35 & 5837,78 & 33,30 \\
\hline $\mathrm{CV}$ & 33,64 & 24,50 & 32,85 & 54,33 & 32,55 & 33,66 & 39,60 & 22,90 & 66,05 & 42,85 & 39,45 \\
\hline $\mathrm{F}$ & $\begin{array}{c}93,52 \\
(32,65)\end{array}$ & $\begin{array}{c}5,42 \\
(21,41)\end{array}$ & $\begin{array}{c}31,97 \\
(12,63)\end{array}$ & $\begin{array}{l}36,27 \\
(8,08)\end{array}$ & $\begin{array}{l}12,90 \\
(8,80)\end{array}$ & $\begin{array}{c}6,04 \\
(11,44)\end{array}$ & $\begin{array}{l}194,29 \\
(17,67)\end{array}$ & $\begin{array}{c}43,18 \\
(10,89)\end{array}$ & $\begin{array}{c}463,81 \\
(1,66)\end{array}$ & $\begin{array}{c}4468,01 \\
(14,61)\end{array}$ & $\begin{array}{l}118,15 \\
(12,87)\end{array}$ \\
\hline ó & 27,85 & 1,82 & 13,14 & 15,83 & 4,15 & 1,90 & 79,89 & 17,25 & 238,52 & 2442,63 & 72,35 \\
\hline $\mathrm{CV}$ & 29,78 & 33,68 & 41,10 & 43,65 & 32,15 & 31,49 & 41,12 & 39,95 & 51,43 & 54,67 & 61,23 \\
\hline G & $\begin{array}{c}37,17 \\
(12,97)\end{array}$ & $\begin{array}{c}2,87 \\
(11,35)\end{array}$ & $\begin{array}{c}56,17 \\
(22,19)\end{array}$ & $\begin{array}{c}83,48 \\
(18,59)\end{array}$ & $\begin{array}{c}20,46 \\
(13,96)\end{array}$ & $\begin{array}{c}6,25 \\
(11,84)\end{array}$ & $\begin{array}{c}107,70 \\
(9,80)\end{array}$ & $\begin{array}{c}81,24 \\
(20,50)\end{array}$ & $\begin{array}{c}269,49 \\
(0,96)\end{array}$ & $\begin{array}{c}6911,42 \\
(22,60)\end{array}$ & $\begin{array}{l}116,71 \\
(12,72)\end{array}$ \\
\hline ó & 11,82 & 1,08 & 14,09 & 27,52 & 6,53 & 1,87 & 30,23 & 20,83 & 87,96 & 2798,39 & 67,22 \\
\hline $\mathrm{CV}$ & 31,80 & 37,51 & 25,09 & 32,96 & 31,90 & 29,99 & 28,07 & 25,63 & 32,64 & 40,49 & 57,60 \\
\hline $\mathrm{R}$ & $\begin{array}{c}49,98 \\
(17,45)\end{array}$ & $\begin{array}{c}5,67 \\
(22,40)\end{array}$ & $\begin{array}{c}30,36 \\
(12,00)\end{array}$ & $\begin{array}{l}113,06 \\
(25,18)\end{array}$ & $\begin{array}{c}19,74 \\
(13,47)\end{array}$ & $\begin{array}{c}9,46 \\
(17,91)\end{array}$ & $\begin{array}{l}220,68 \\
(20,07)\end{array}$ & $\begin{array}{c}97,28 \\
(24,54)\end{array}$ & $\begin{array}{c}25782,64 \\
(92,28)\end{array}$ & $\begin{array}{c}2090,67 \\
(6,83)\end{array}$ & $\begin{array}{l}335,49 \\
(36,56)\end{array}$ \\
\hline ó & 14,82 & 1,45 & 20,91 & 34,23 & 0,49 & 1,45 & 144,33 & 45,43 & 30039,40 & 168,80 & 101,01 \\
\hline $\mathrm{CV}$ & 29,66 & 25,58 & 68,87 & 30,28 & 2,50 & 15,35 & 65,40 & 46,70 & 116,51 & 8,07 & 30,11 \\
\hline Total & $\begin{array}{c}286,47 \\
(100)\end{array}$ & $\begin{array}{l}25,30 \\
(100)\end{array}$ & $\begin{array}{c}253,08 \\
(100)\end{array}$ & $\begin{array}{c}449,02 \\
(100)\end{array}$ & $\begin{array}{c}146,52 \\
(100)\end{array}$ & $\begin{array}{l}52,81 \\
(100)\end{array}$ & $\begin{array}{c}1099,50 \\
(100)\end{array}$ & $\begin{array}{c}396,37 \\
(100)\end{array}$ & $\begin{array}{c}27939,89 \\
(100)\end{array}$ & $\begin{array}{c}30587,86 \\
(100)\end{array}$ & $\begin{array}{c}917,74 \\
(100)\end{array}$ \\
\hline
\end{tabular}

${ }^{1} \mathrm{Mt}=$ madeira do tronco; $\mathrm{Ct}=$ casca do tronco; $\mathrm{F}=$ folhas; $\mathrm{G}=$ galhos; $\mathrm{R}=$ raiz; ${ }^{12}$ Valores entre parênteses referem-se à contribuição percentual de cada componente em relação ao total de cada nutriente analisado; ${ }^{13}$ ó = Desvio padrão; ${ }^{\vee 4} \mathrm{CV}=\operatorname{coeficiente}$ de variação.

Tabela 4 - Estimativa da remoção de nutrientes através da colheita da biomassa de Eucalyptus dunnii aos 4 anos de idade. Table 4 - Estimation of nutrients removal by harvesting the biomass of Eucalyptus dunnii at 4 years of age.

\begin{tabular}{|c|c|c|c|c|c|c|c|c|c|c|c|}
\hline \multirow{2}{*}{$\begin{array}{l}\text { Cenário de } \\
\text { Colheita }\end{array}$} & \multicolumn{6}{|c|}{ Macronutrientes $\left(\mathrm{kg} \mathrm{ha}^{-1}\right)$} & \multicolumn{5}{|c|}{ Micronutrientes $\left(\mathrm{g} \mathrm{ha}^{-1}\right)$} \\
\hline & $\mathrm{N}$ & $\mathrm{P}$ & $\mathrm{K}$ & $\mathrm{Ca}$ & $\mathrm{Mg}$ & $\mathrm{S}$ & B & $\mathrm{Cu}$ & $\mathrm{Fe}$ & Mn & $\mathrm{Zn}$ \\
\hline $1^{\backslash 1}$ & 237 & 20 & 223 & 336 & 127 & 43 & 879 & 299 & 2157 & 28497 & 582 \\
\hline 2 & 106 & 11 & 135 & 216 & 93 & 31 & 577 & 175 & 1424 & 17118 & 347 \\
\hline 3 & 73 & 6 & 99 & 36 & 50 & 26 & 443 & 133 & 1234 & 3495 & 263 \\
\hline
\end{tabular}

${ }^{1} 1$ = Colheita de toda árvore; 2 = Colheita da madeira com casca; 3 = Colheita da madeira;

al. (2008b) em um estudo de E. urograndis e E. grandis aos 4,5 anos de idade em diferentes regiões do Brasil; por Schumacher e Caldeira (2001), estudando E. globulus aos 4 anos de idade; e por Beulch (2013), avaliando E. saligna com 4 anos de idade, no Rio Grande do Sul. Turner e Lambert (2008), em estudo sobre E. grandis e E. pilularis com 5 anos de idade, encontraram produção de biomassa consideravelmente inferior, sendo 52,23 e $35,13 \mathrm{Mg} \mathrm{ha}^{-1}$.

Fatores genéticos (melhoramento e procedências), edafoclimáticos e de manejo estão relacionados diretamente à capacidade de produção das espécies, porém Barros e Comerford (2002) explicaram que a grande variabilidade da produtividade das plantações com o eucalipto nas diferentes regiões está associada, principalmente, aos diversos tipos de solos que apresentam teores disponíveis e totais de nutrientes numa faixa bastante ampla.

Para a partição relativa da biomassa acima do solo, valores similares foram encontrados por Poggiani et al. (1983) e Pereira et al. (1984) em Eucalytpus saligna aos 8 e 9 anos de idade, sendo identificado que $85 \%$

Revista Árvore, Viçosa-MG, v.39, n.5, p.873-882, 2015 
da biomassa aérea se encontrava nos componentes madeira do tronco + casca do tronco; Spangenberg et al. (1996), em Eucalyptus urograndis aos 4,5 anos de idade, identificaram que $90 \%$ da biomassa está distribuída nesses mesmos componentes. Viera (2012) e Beulch (2013) identificaram, em estudos com E. globulus aos 10 anos e E. saligna aos 4 anos de idade, que os componentes madeira + casca do tronco representavam $94 \%$ e $86 \%$ da biomassa acima do solo, respectivamente. Entretanto, alguns autores obtiveram resultados diferentes: Schumacher(1998), em E. globulus aos 4 anos de idade, observou que a copa representava $28 \%$ da biomassa total acima do solo, enquanto os $72 \%$ restantes eram representados pela madeira do tronco + casca do tronco. Esse mesmo autor encontrou a mesma tendência de distribuição em um estudo sobre $E$. saligna aos 4 e 7 anos de idade, o que também foi observado por Caldeira (1998) em Acacia mearnsii aos 2 anos e aos 4 meses de idade. Turner e Lambert (2008), em estudo com E. grandis e E. pilularis aos 5 anos de idade, verificaram que, em biomassa acima do solo, 70 e $73 \%$ eram representados pela biomassa do tronco + casca do tronco.

Quanto aos teores dos nutrientes, embora as componentes madeira do tronco e raiz representassem a maior parte da biomassa acumulada, a concentração dos macronutrientes nelas é menor, porém, quanto aos micronutrientes, se observou que essa tendência se inverteu. Na componente folha está concentrada a maior parte dos nutrientes N (64\%), P (46\%), K (34\%) e S (38\%), exceto Ca (49\%), Mg (45\%) e Mn (44\%), em que os maiores teores estão na casca do tronco e no Fe $(90 \%)$, que se encontra na raiz.

A tendência é de que a maioria dos nutrientes se concentre nas estruturas mais novas da planta, principalmente nas folhas, onde ocorrem os principais processos metabólicos (transpiração e fotossíntese) (VIERA, 2012).

A alta concentração de Ca na casca está relacionada à baixa mobilidade desse elemento no floema das plantas e por ser também componente estrutural da membrana celular (BRUN, 2004). Quanto a K e S, os maiores teores foram observados na madeira do tronco, seguidos pelo P. Os altos teores de $\mathrm{Fe}$ na raiz podem estar relacionados à contaminação por óxidos de Fe do solo (HOPPE, 2003).

De acordo com Pallardy (2008), a diferença na concentração de nutrientes, entre os componentes da planta, está intrinsecamente relacionada à ciclagem bioquímica, em que, com a idade, nutrientes de tecidos senescentes tendem a se deslocar para regiões com maior atividade metabólica. Nesse mesmo contexto, Poggiani e Schumacher (2004) destacaram que a ciclagem bioquímica apresenta maior importância para a manutenção dos nutrientes com alta mobilidade $(\mathrm{N}, \mathrm{P}, \mathrm{K}$ e $\mathrm{Mg})$ e menor dos nutrientes pouco móveis $(\mathrm{Ca}, \mathrm{S})$ e micronutrientes.

Segundo Hernandéz et al. (2009), as concentrações dos nutrientes nos diversos componentes das árvores de eucalipto, bem como a sua produção de biomassa, têm relação direta com a densidade de plantio e a fertilidade do solo. Além desses fatores, Poggiani e Schumacher (1993) destacaram que as características nutricionais das espécies e a idade de corte influenciavam o acúmulo de nutrientes por compartimento na biomassa arbórea.

\subsection{Remoção de nutrientes pela colheita da biomassa}

As maiores diferenças nas taxas de devolução de nutrientes de acordo com a intensidade de colheita da biomassa encontram-se nos elementos Ca e $\mathrm{Mg}$, destacando-se que esses nutrientes se apresentam em maiores proporções na componente casca. Segundo Schumacher e Caldeira (2001), a colheita da madeira com a casca potencializa a remoção de nutrientes do sítio florestal, principalmente $\mathrm{Ca}$. Considerando a colheita da madeira com a casca e apenas a madeira do tronco, comportamento semelhante foi encontrado por Viera (2012) e Neves (2000), em estudos com o híbrido de Eucalytpus urophylla $\mathrm{x}$ Eucalytpus globulus e com Eucalytpus grandis e Eucalytpus saligna. Porém, a mesma tendência de distribuição não foi encontrada por Spangenberg et al. (1996) e Merino et al. (2005), estudando Eucalytpus urograndis e Eucalytpus globulus, respectivamente.

A colheita de toda a biomassa acima do solo se apresenta como o cenário mais agressivo de remoção dos nutrientes, o que, consequentemente, se reflete no balanço nutricional e na manutenção da capacidade produtiva do sítio.

Apesar de existir maior exaustão dos nutrientes do sistema com o aumento da intensidade da colheita da biomassa, na prática o esgotamento absoluto dos nutrientes não acontece. Ao analisar esse comportamento, observa-se transição de um nível de

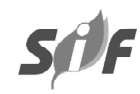

Revista Árvore, Viçosa-MG, v.39, n.5, p.873-882, 2015 
produtividade em um ciclo, para um nível de produtividade inferior no ciclo seguinte, e assim sucessivamente (WITSCHORECK, 2008).

Os sítios mais produtivos, por extraírem maiores quantidades de nutrientes a cada rotação, tendem a atingir a exaustão mais rapidamente, indicando que a manutenção de níveis elevados de produtividade dependerá do uso de fertilizantes, baixa intensidade de colheita da biomassa e princípios conservacionistas (BIZON, 2005).

Destaca-se que essa simulação está sendo feita para um povoamento aos 4 anos e não aos 7 ; $\log$ o, a dinâmica nutricional deverá se alterar. Portanto, dependendo do nível de remoção dos nutrientes, pela colheita da biomassa, grandes quantidades de fertilizantes (aumento dos custos operacionais) poderão ser utilizadas para reestabelecer o equilíbrio nutricional e garantir a produtividade florestal nos próximos ciclos de produção.

\section{CONCLUSÕES}

Na madeira do tronco, verificou-se a maior produção de biomassa e os maiores estoques dos nutrientes $\mathrm{K}$, $\mathrm{Mg}, \mathrm{S}, \mathrm{B}$ e $\mathrm{Cu}$. O maior estoque de $\mathrm{N}$ foi observado na folha, de Ca e Mn na casca e Fe e $\mathrm{Zn}$ na raiz.

Entre os três cenários de colheita avaliados, $\mathrm{Ca}$ e $\mathrm{K}$ foram os elementos que apresentaram o maior risco para a manutenção da produtividade.

A colheita apenas da madeira poderá contribuir sistematicamente para a sustentabilidade nutricional do sítio.

\section{AGRADECIMENTOS}

A Stora Enso Florestal RS, pelo apoio logístico e financeiro para a realização deste trabalho.

\section{REFERÊNCIAS}

ABRAF. Anuário Estatístico 2013. Acessado em 8 out. 2013. Disponível em: http:// www.abraflor.org.br/estatisticas/ABRAF13/ ABRAF13-BR.pdf.

BARROS, N.F.; COMERFORD, N.B. Sustentabilidade da produção de florestas plantadas na região tropical. Tópicos em Ciência do Solo, v.2, p.487-592, 2002.
BARROS, N.F.; NOVAIS, R.F. Fertilização e correção do solo para o plantio de eucalipto. In: BARROS, N.F; NOVAIS, R.F. Relação Solo Eucalipto. Viçosa, MG: Folha de Viçosa, 1990. p.127-181.

BEULCH, L.S. Biomassa e nutrientes em um povoamento de Eucalyptus saligna Smith submetido ao primeiro desbaste. 2013. 58f. Dissertação (Mestrado em Engenharia Florestal) - Universidade Federal de Santa Maria, Santa Maria, 2013.

\section{BIZON, J.M.C. Avaliação da sustentabilidade nutricional de plantios de Pinus taeda L. usando um balanço de entrada-saída de nutrientes. 2005. 95f. Dissertação (Mestrado em Recursos Florestais) - Escola Superior de Agricultura Luiz de Queiroz, Piracicaba, 2005.}

BRUN, E.J. Biomassa e nutrientes na floresta estacional decidual, em Santa Tereza, RS. 2004. 136f. Dissertação (Mestrado em Engenharia Florestal) - Universidade Federal de Santa Maria, Santa Maria, 2004.

CALDEIRA, M.V.W. Quantificação da biomassa e do conteúdo de nutrientes em diferentes procedências de Acácianegra (Acacia mearnsii De Wild.) 1998. 96f. Dissertação (Mestrado em Engenharia Florestal) Universidade Federal de Santa Maria, Santa Maria, 1998.

COMISSÃO DE QUÍMICA E FERTILIDADE DO SOLO - CQFSRS/SC. Manual de adubação e calagem para os estados do Rio Grande do Sul e Santa Catarina. 10.ed. Porto Alegre: Sociedade Brasileira de Ciência do Solo/Comissão de Química e Fertilidade do Solo, 2004. 400p.

EMPRESA BRASILEIRA DE PESQUISA AGROPECUÁRIA - EMBRAPA. Centro Nacional de Pesquisa de Solos. Manual de métodos de análise de solos. 2.ed. Rio de Janeiro: 1997.212p.

EMPRESA BRASILEIRA DE PESQUISA AGROPECUÁRIA - EMBRAPA. Centro Nacional de Pesquisa de Solos. Sistema brasileiro de classificação de solos. Rio de Janeiro: 2006. 306p. 
HERNÁNDEZ, J.; PINO, A.; SALVO, L.; ARRARTE. G. Nutrient export and harvest residue decomposition patterns of a Eucalyptus dunnii Maiden plantation in temperate climate of Uruguay. Forest Ecology and

Management, v.258, n.2, p.92-99, 2009.

HIGA, R.C.V. Avaliação e recuperação de Eucalyptus dunnii Maiden atingidos por geadas em campo Tenente, Paraná. 1998. 100f. Tese (Doutorado em Ciências Florestais) - Universidade Federal do Paraná, Curitiba, 1998.

HOPPE, J.M. Biomassa e nutrientes em Platanus $x$ acerifolia (Aiton) Willd. estabelecido no município de Dom Feliciano - RS. 2003.143f. Tese (Doutorado em Engenharia Florestal) - Universidade de Santa Maria, Santa Maria, 2003.

JOVANOVIC, T.; ARNOLD, R.; BOOTH, T. Determining the climatic suitability of Eucalyptus dunniifor plantations in Australia, China and Central and South America. New Forests, v.19, n.3, p.215-226, 2000.

MALUF, J.R.T. Nova classificação climática do Estado do Rio Grande do Sul. Revista Brasileira de Agrometeorologia, v.8, n.1, p.141-150, 2000.

MERINO, A.; BALBOA, M.A.; SOALLEIRO, R.R.; GONZÁLES, A. J. G. Nutrient exports under different harvesting regimes in fast-growing forest plantations in southern Europe. Forest Ecology and Management, v.207, n.3, p.325-339, 2005.

NEVES, J.C.L. Produção e partição de biomassa, aspectos nutricionais e hídricos em plantios clonais de eucalipto na região litorânea do Espírito Santo. 2000. 191f. Tese (Doutorado em Produção Vegetal) - Universidade Estadual do Norte Fluminense, Campos dos Goytacazes, 2000.

PALLARDY, S. Physiology of woody plants. San Diego: Academic Press, 2008. 454p.

PEREIRA, A.R.; ANDRADE, D.C.; LEAL, P.G.L Produção de biomassa e remoção de nutrientes em povoamentos de Eucalyptus citriodora e Eucalyptus saligna cultivados na região de cerrado de Minas Gerais. Floresta. v. 15, n.1, p.18-16, 1984.

POGGIANI, F.; COUTO, H.T.Z.; CORRADINI, L. et al. Exploração de biomassa e nutrientes através da exportação dos troncos e das copas de um povoamento de Eucalyptus saligna. IPEF, v. 25, p. 37-39, 1983.

POGGIANI, F.; SCHUMACHER, M.V.

Exportação de biomassa e nutrientes através da exploração dos troncos e das copas de um povoamento de Eucalyptus saligna. IPEF, n.25, p.37-39, 1993.

POGGIANI, F.; SCHUMACHER, M.V. Nutrient cycling in native forest. In: GONÇALVES, J.L.M.; BENEDETTI, V. Forest nutrition and fertilization. Piracicaba: IPEF, 2004. p.285-305.

POSCHENRIEDER, C.; GUNSE, B.; CORRALES, I.; BARCELO, I. A glance into aluminum toxicity and resistance in plants. Science of the Total Environment, v.400, n.1-3, p.356-368, 2008.

RYAN, M. G.; STAPE, J. L.; BINKLEY, D.; FONSECA, S.; LOOS, R. A.; TAKAHASHI, E. N.; SILVA, C. R.; SILVA, S. R.; HAKAMADA R. E.; FERREIRA, J. M.; LIMA, A. M. N.; GAVA, J. L.; LEITE, F. P.; ANDRADE, H. B.; ALVES, J. M.; SILVA, G. G. C. S. Factors controlling Eucalyptus productivity: How water availability and stand structure alter production and carbon allocation. Forest Ecology and

Management, v.259, n.9, p.1695-1703, 2010.

RIBEIRO, A.C.; GUIMARÃES, P.T.G.; ALVAREZ V., V.H. Recomendação para o uso de corretivos e fertilizantes em Minas Gerais - $5^{\mathbf{a}}$ aproximação. Viçosa, MG: Universidade Federal de Viçosa, 1999. 359p.

SANTANA, R. C.; BARROS, N. F.; NOVAIS, R.F.; LEITE, H. G.; COMEFORD, N. B. Alocação de nutrientes em plantios de eucalipto no Brasil. Revista Brasileira de Ciência do Solo, v.32, p.2723-2733, 2008a.Numero Especial

SANTANA, R. C.; BARROS, N. F.; LEITE, H. G.; COMEFORD, N. B.; NOVAIS, R.F. Estimativa de

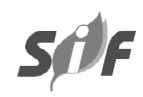

Revista Árvore, Viçosa-MG, v.39, n.5, p.873-882, 2015 
biomassa de plantios de eucalipto no Brasil. Revista Árvore, Viçosa, v. 32, n. 4, p. 697-706, jul./ago. 2008b.

SAS. Statistical analysis system: Programa de computador, ambiente VM. Cary: 2003. Versão 6.08 .

SILVA, I.R.; NOVAIS, R. F.; JHAM, G. N.; BARROS, N. F.; GEBRIM, F. O.; NUNES, F. N.; NEVES, J. C. L.; LEITE, F. P. Responses of eucalypt species to aluminum: the possible involvement of low molecular weight organic acids in the Al tolerance mechanism. Tree Physiology, v.24, p.1267-1277, 2004.

STRECK, E. V.; KÄMPF, N.; DALMOLIN, R. S. D.; KLAMT, E.; NASCIMENTO, P. C.; SCHNEIDER, P.; GIASSON, E.; PINTO L. F. S. Solos do Rio Grande do Sul. Porto Alegre: UFRGS/ EMATER/RS, 2008. 107p.

SCHUMACHER, M.V. Estudo da biomassa e dos nutrientes de um povoamento de Eucalyptus globulus (Labillardière) sub-espécie bicostata. Revista Árvore, v.22, n.2, p.281-286, 1998.

SCHUMACHER, M.V.; CALDEIRA, M.V.W. Estimativa da biomassa e do conteúdo de nutrientes de um povoamento de Eucalyptus globulus (labillardière) sub-espécie maidenii. Ciência Florestal, v. 11, n.1, p.45-53, 2001.

SCHUMACHER, M.V.; POGGIANI, F. Retorno de nutrientes via deposição de serapilheira em um povoamento de acácia - negra (Acacia mearnsii De Wild.) no estado do Rio Grande do Sul.

Revista Árvore, v.27, n.1, p.29-37, 2003.

SPANGENBERG, A.; GRIM, U.; SILVA, J.R.S.; FOLSTER, H. Nutrient store and export rates of Eucalyptus urograndis plantations in eastern Amazonia (Jari). Forest Ecology and Management, v.80, p.225-234, 1996.

TAHARA, K.; NORISADA, M.; YAMANOSHITA, T.; KOJIMA, K. Role of aluminum-binding ligands in aluminum resistance of Eucalyptus camaldulensis and Melaleuca cajuputi. Plant and Soil, v.302, n.1-2, p.175-187, 2008.

TEDESCO, M.J.; GIANELLO, C. BISSANI, C.A.; BOHNEN, H.; VOLKWEISS, S.J. Análise de solo, plantas e outros materiais. 2.ed. Porto Alegre: Departamento de Solos, UFRGS, 1995. 174p. (Boletim Técnico, 5).

TURNER, J.; LAMBERT, M.J. Nutrient cycling in age sequences of two Eucalyptus plantation species. Forest Ecology and Management, v.255, p.1701-1712, 2008.

VIERA, M.; SCHUMACHER, M.V. Deposição de serapilheira e de macronutrientes em um povoamento de acácia-negra (Acacia mearnsii De Wild.) no Rio Grande do Sul. Ciência Florestal, v.20, n.2, p.225-233, 2010.

VIERA, M. Dinâmica nutricional em um povoamento híbrido de Eucalyptus urophylla $\mathrm{x}$ Eucalyptus globulus em Eldorado do Sul-RS, Brasil. 2012. 119p. Tese (Doutorado em Engenharia Florestal) Universidade Federal de Santa Maria, Santa Maria, 2012.

VIEIRA, F.C.B.; ZHENLI, L. H.; BAYER, C.; STOFFELLA, P. J.; BALIGAR, V. C. Organic amendment effect on the transformation and fractionation of aluminum in acidic sandysoil. Communications in Soil Science and Plant Analysis, v.39, p. 2678-2694, 2008.

VITAL, A. R. T.; GUERRINI, I. A.; FRANKEN, W. K.; FONSECA, R. C .B. Produção de serapilheira e ciclagem de nutrientes de uma floresta estacional semidecidual em zona ripária. Revista Árvore, v.28, n.6, p.793-800, 2004.

WITSCHORECK, R. Biomassa e nutrientes no corte raso de um povoamento de Pinus taeda 1 . de 17 anos de idade no município de cambará do sul-RS. 2008. 80f. Dissertação (Mestrado em Engenharia Florestal) - Universidade Federal de Santa Maria, Santa Maria, 2008. 